\title{
Unique reducibility of multiple blocking sets
}

\author{
Nóra V. Harrach*
}

\begin{abstract}
A weighted $t$-fold $(n-k)$-blocking set $B$ of $\operatorname{PG}(n, q)$ always contains a minimal weighted $t$-fold $(n-k)$-blocking set. We prove that, if $|B|<(t+1) q^{n-k}+\theta_{n-k-1}$, then the minimal weighted $t$-fold $(n-k)$-blocking set contained in $B$ is unique.
\end{abstract}

Mathematics Subject Classification (2010). 51E21, 51E20.

Keywords. Projective space, multiple blocking sets.

\section{Introduction}

A $t$-fold $(n-k)$-blocking set of $\operatorname{PG}(n, q)$ is a set of points which meets every $k$-dimensional subspace in at least $t$ points. To exclude the trivial cases we will always suppose that $0<k<n$. If the points of the set are not all different, so the set is a multiset of points, then it is called a weighted $t$-fold $(n-k)$-blocking set. A weight function of $\operatorname{PG}(n, q)$ is a mapping from the point set of $\mathrm{PG}(n, q)$ to the set of nonnegative integers. For a point $P$ the integer $w(P)$ is the weight of $P$. There is a natural correspondence between multisets and weight functions of $\mathrm{PG}(n, q)$ : let the weight of a point be the multiplicity of that point in the set. For a weight function $w$, the weight of a set $M$ of points is by definition the sum of the weights of all its points, denoted by $w(M)$, and $w(\mathrm{PG}(n, q))=:|w|$ can be called the total weight of $w$. The multiset associated to a weight function $w$ is a $t$-fold $(n-k)$-blocking set if and only if the weight of every $k$-dimensional subspace is at least $t$. If this is the case, then we will call the weight function $w$ a $t$-fold $(n-k)$-blocking set for short.

*The author was supported by OTKA Grant K81310. 
If $w$ is a $t$-fold $(n-k)$-blocking set, then a point $P$ is called a non-essential point of $w$, if the weight of every $k$-subspace containing $P$ is at least $t+1$ and $w(P) \geq 1$. Then the weight function $w^{\prime}$ defined by

$$
w^{\prime}(Q)= \begin{cases}w(Q) & \text { if } Q \neq P \\ w(P)-1 & \text { if } Q=P\end{cases}
$$

is also a $t$-fold $(n-k)$-blocking set.

If $w$ and $w^{\prime}$ are weight functions, and $w^{\prime}(P) \leq w(P)$ for all points $P \in$ $\operatorname{PG}(n, q)$, then we will say that $w^{\prime}$ is contained in $w$, and denote this by $w^{\prime} \leq w$.

The $t$-fold $(n-k)$-blocking set $w$ is said to be minimal if $w^{\prime} \equiv w$ for any $t$-fold $(n-k)$-blocking set $w^{\prime}$ contained in $w$.

A $t$-fold $(n-k)$-blocking set is not minimal if and only if it has non-essential points. If we start reducing the weight of the non-essential points one by one, always checking carefully that the resulting set/weight function is still a $t$-fold $(n-k)$-blocking set, then after some steps we will arrive at a minimal $t$-fold $(n-k)$-blocking set. It is a natural question to ask if there are conditions which guarantee the uniqueness of this minimal $t$-fold $(n-k)$-blocking set. Here, two weight fuctions $w^{\prime}$ and $w^{\prime \prime}$ are considered to be different if there is a point $P$, such that $w^{\prime}(P) \neq w^{\prime \prime}(P)$.

In [12] such a condition is given for non-weighted 1-fold 1-blocking sets of $\operatorname{PG}(2, q)$.

Result 1.1. (Szönyi, [12]) A non-weighted 1-fold 1-blocking set of $\mathrm{PG}(2, q)$, with size smaller than $2 q+1$ contains a unique minimal 1-fold 1-blocking set.

This result was recently generalized to non-weighted 1-fold $(n-k)$-blocking sets of $\mathrm{PG}(n, q)$ in $[9]$.

Result 1.2. (Lavrauw, Storme and Van de Voorde, [9]) A non-weighted 1fold $(n-k)$-blocking set of $\mathrm{PG}(n, q)$, with size smaller than $2 q^{n-k}$ contains a unique minimal 1-fold $(n-k)$-blocking set.

Using the standard notation $\theta_{m}=\frac{q^{m+1}-1}{q-1}$ for the number of points of an $m$-dimensional subspace of $\mathrm{PG}(n, q)$, our result is the following. 
Theorem 1.3. A weighted $t$-fold $(n-k)$-blocking set of $\mathrm{PG}(n, q)$, with total weight smaller than

$$
(t+1) q^{n-k}+\theta_{n-k-1}
$$

contains a unique minimal weighted $t$-fold $(n-k)$-blocking set.

Note that Theorem 1.3 is stronger than Result 1.2. Examples in the last section show that the bound is sharp if $t=1$, or if $k=n-1$.

\section{$2 t$-fold $(n-k)$-blocking sets containing two minimal $t$-fold $(n-k)$-blocking sets}

Let $w$ be a $t$-fold $(n-k)$-blocking set. We will now define a new weight function $s_{w}$ on the points of $\mathrm{PG}(n, q)$. For a point $P$ let $s_{w}(P)$ be the largest integer for which the weight function $w^{\prime}$ defined by

$$
w^{\prime}(Q)= \begin{cases}w(Q) & \text { if } Q \neq P \\ w(P)-s_{w}(P) & \text { if } Q=P\end{cases}
$$

is also a $t$-fold $(n-k)$-blocking set. Then $w(P) \geq s_{w}(P) \geq 0$, so if $w(P)=0$, then $s_{w}(P)=0$. It is also clear that $w$ is minimal if and only if $s_{w} \equiv 0$.

Lemma 2.1. For a t-fold $(n-k)$-blocking set $w$ and $P \in \operatorname{PG}(n, q)$ the following are true:

(a) $s_{w}(P)=\min \left\{w(P), \min _{P \in \Pi_{k}}\left(w\left(\Pi_{k}\right)-t\right)\right\}$, where $\Pi_{k}$ runs along the $k$ dimensional subspaces containing $P$;

(b) $s_{w}(P)=\max _{w^{\prime} \leq w}\left\{w(P)-w^{\prime}(P)\right\}$, where $w^{\prime}$ runs along the $t$-fold $(n-k)$ blocking sets contained in $w$.

Lemma 2.2. If $w$ is a t-fold $(n-k)$-blocking set which contains two different minimal $t$-fold $(n-k)$-blocking sets, then there is a weight function $v \leq w$ and a line $l^{*}$ with the following properties:

(a) $v\left(\Pi_{k}\right) \geq t$ for any $k$-subspace $\Pi_{k}$ not containing $l^{*}$; 
(b) $v\left(\Pi_{k}\right) \geq t-1$ for any $k$-subspace $\Pi_{k}$ containing $l^{*}$;

(c) there is a $k$-subspace $\Pi_{k}^{*}$ containing $l^{*}$, for which $v\left(\Pi_{k}^{*}\right)=t-1$;

(d) $|w| \geq|v|+2$.

Proof. Let $w^{\prime}$ and $w^{\prime \prime}$ be two different minimal $t$-fold $(n-k)$-blocking sets contained in $w$. Then there is a point $P^{*} \in \operatorname{PG}(n, q)$, such that $w^{\prime}\left(P^{*}\right)>$ $w^{\prime \prime}\left(P^{*}\right)$. Define $\tilde{w}$ as follows:

$$
\tilde{w}(Q)= \begin{cases}w(Q) & \text { if } Q \neq P^{*} \\ w^{\prime}\left(P^{*}\right) & \text { if } Q=P^{*}\end{cases}
$$

Then $\tilde{w}$ is a $t$-fold $(n-k)$-blocking set, $w^{\prime}, w^{\prime \prime} \leq \tilde{w}$, and Lemma 2.1(b) yields that $s_{\tilde{w}}\left(P^{*}\right) \geq \tilde{w}\left(P^{*}\right)-w^{\prime \prime}\left(P^{*}\right)=w^{\prime}\left(P^{*}\right)-w^{\prime \prime}\left(P^{*}\right)>0$.

As $\tilde{w}$ contains the minimal $t$-fold $(n-k)$-blocking set $w^{\prime}$, we can start reducing the weight of the points with $\tilde{w}(P)>w^{\prime}(P)$, one at a time, until we arrive at $w^{\prime}$. Formally, let $\tilde{w}=w_{1} \geq w_{2} \geq \cdots \geq w_{m}=w^{\prime}$ be a sequence of $t$-fold $(n-k)$-blocking sets, such that for $i \in\{1,2, \ldots, m-1\}$ the $t$-fold $(n-k)$-blocking sets $w_{i}$ and $w_{i+1}$ only differ in one point $P_{i}$, and $w_{i+1}\left(P_{i}\right)=$ $w_{i}\left(P_{i}\right)-1$. Clearly $P_{i} \neq P^{*}$, and the points $P_{i}$ are not necessarily all different. It is also clear that $\tilde{w} \neq w^{\prime}$, because $\tilde{w}=w^{\prime}$ would mean that $w^{\prime \prime}$ is contained in $w^{\prime}$, which is a contradiction, so $m \geq 2$ follows.

By Lemma 2.1(a), $s_{w_{i+1}} \leq s_{w_{i}}$, in fact, for any point $Q$, either $s_{w_{i+1}}(Q)=$ $s_{w_{i}}(Q)$, or $s_{w_{i+1}}(Q)=s_{w_{i}}(Q)-1$. For the point $P^{*}$ we have $s_{\tilde{w}}\left(P^{*}\right)>0$ by $\left(^{*}\right)$, and $s_{w^{\prime}}\left(P^{*}\right)=0$ by the minimality of $w^{\prime}$. So there will be an $i \in\{1,2, \ldots, m-$ $1\}$ such that $s_{w_{i}}\left(P^{*}\right)=1$ and $s_{w_{i+1}}\left(P^{*}\right)=0$. The weight functions $w_{i}$ and $w_{i+1}$ only differ in the point $P_{i}$. Then by Lemma 2.1 (a) there is a $k$-space $\Pi_{k}^{*}$ which contains $P_{i}$ and $P^{*}$, and has weight $w_{i}\left(\Pi_{k}^{*}\right)=t+1$. Also by Lemma 2.1(a) this yields $s_{w_{i}}\left(P_{i}\right) \leq 1$, and as $w_{i+1}\left(P_{i}\right)=w_{i}\left(P_{i}\right)-1$, so $P_{i}$ is a nonessential point of $w_{i}$, then $s_{w_{i}}\left(P_{i}\right)=1$ follows. Thus for any $k$-dimensional subspace $\Pi_{k}$, which contains $P^{*}$ and/or $P_{i}$ we have $w_{i}\left(\Pi_{k}\right) \geq t+1$.

Let $l^{*}$ be the line connecting $P_{i}$ and $P^{*}$, and define $v$ to be the following weight function:

$$
v(Q)= \begin{cases}w_{i}(Q) & \text { if } Q \notin\left\{P^{*}, P_{i}\right\} \\ w_{i}(Q)-1 & \text { if } Q \in\left\{P^{*}, P_{i}\right\}\end{cases}
$$


Clearly $|w| \geq\left|w_{i}\right|=|v|+2$, and $v$ is a weight function contained in $w$. The weight of a $k$-subspace $\Pi_{k}$ is $w_{i-1}\left(\Pi_{k}\right)-\left|\Pi_{k} \cap\left\{P^{*}, P_{i}\right\}\right|$. Thus, $v, l^{*}$ and $\Pi_{k}^{*}$ satisfy the properties given in the lemma.

\section{$3 \quad t$-fold nuclei}

If $t=1, n=2, k=1$, then Lemma 2.2 yields that if $w$ is a 1-fold 1-blocking set of $\mathrm{PG}(2, q)$ containing two different minimal 1-fold 1-blocking sets, then $w$ contains a weight function $v$, which defines a blocking set of the affine plane $\mathrm{AG}(2, q):=\mathrm{PG}(2, q) \backslash l^{*}$. Thus $|w(\mathrm{PG}(2, q))| \geq s(q)+2$, where $s(q)$ denotes the size of the smallest 1-blocking set of $\operatorname{AG}(2, q)$. There are several independent proofs for $s(q)=2 q-1$, from which Result 1.1 follows (see Jamison [8], Brouwer and Schrijver [5], Blokhuis [2], Szőnyi [12]).

In [2], $s(q)=2 q-1$ is proved as a corollary of a theorem on nuclei of point sets. Now we generalize the notion of nucleus to multisets/weight functions.

Definition 3.1. (1) Let $S$ be a multiset of $\mathrm{PG}(n, q)$. A point $P \notin S$ will be called a $t$-fold nucleus of $S$ if every line through $P$ meets $S$ in at least $t$ points, counted with multiplicities.

(2) Let $w$ be a weight function of $\operatorname{PG}(n, q)$. A point $P \in \operatorname{PG}(n, q)$ with $w(P)=0$ will be called a $t$-fold nucleus of $w$ if every line through $P$ has weight at least $t$.

For $S$ to have nuclei, clearly $|S| \geq t \theta_{n-1}$ is needed. Let $|S|=t \theta_{n-1}+r, r \geq 0$.

Note that for $|S|=t \theta_{n-1}-r, r \geq 0$, a 'symmetric' version of the definition can be: a point $P \notin S$ is a $t$-fold nucleus of $S$, if every line through $P$ meets $S$ in at most $t$ points, counted with multiplicities.

The notion of nucleus was first introduced by Mazzocca for affine sets for $n=2, t=1$ and $r=0$. Blokhuis extended the notion to $r \geq 0$ in [2] and to $t \geq 1$ in [3], and Sziklai generalized the definition for sets of the projective space $\mathrm{PG}(n, q)$ in [11]. (The 'symmetric' version was introduced in [7] and [11].)

Denote by $N^{t}(S)$ the set of $t$-fold nuclei of $S$, and let $p$ be the characteristic of the field $\mathrm{GF}(q)$. 
Result 3.2. (Sziklai, [11]) Let $S$ be a set of points in $\operatorname{PG}(n, q)$ with $|S|=$ $t \theta_{n-1}+r, r \geq 0$. Let $H_{\infty}$ be a given hyperplane, $\left|S \cap H_{\infty}\right|=m_{\infty}$. Then

$$
\left|N^{t}(S) \backslash H_{\infty}\right| \leq(r+1)(q-1),
$$

provided that $\left(\begin{array}{c}t \theta_{n-1}+r-m_{\infty} \\ r+1\end{array}\right) \neq 0(\bmod p)$.

Result 3.2 was proved in the case when $m_{\infty}=0, n=2$ by Blokhuis and Wilbrink $(r=0, t=1$, see [4]) and by Blokhuis (for $r \geq 0, t=1$, see [2], and for $r \geq 0, t \geq 1$ see [3]). The 'symmetric' version was also settled by Sziklai in $[11]$.

As Result 3.2 is not applicable when $\left(\begin{array}{c}t \theta_{n-1}+r-m_{\infty} \\ r+1\end{array}\right)=0(\bmod p)$, to obtain an upper bound in this case, Ball presented the following theorem.

Result 3.3. (Ball, [1]) Let $S$ be a set of points in $\operatorname{PG}(n, q)$ with $|S|=$ $t \theta_{n-1}+r, r \geq 0$, and let $H_{\infty}$ be a given hyperplane, $\left|S \cap H_{\infty}\right|=m_{\infty}$. Then

$$
\left|N^{t}(S) \backslash H_{\infty}\right| \leq(r+1+j)(q-1),
$$

provided that the binomial coefficient

$$
\left(\begin{array}{c}
t \theta_{n-1}+r-m_{\infty} \\
r+1+j
\end{array}\right) \neq 0 \quad(\bmod p)
$$

for some $j \geq 0$.

The proof of Result 3.2 and 3.3 can be easily copied for multisets/weight functions and we obtain the following lemma.

Lemma 3.4. Let $w$ be a weight function on $\mathrm{PG}(n, q)$ and $H_{\infty}$ a given hyperplane with $w\left(H_{\infty}\right)=m_{\infty}$. Suppose that $w(\mathrm{PG}(n, q))=t \theta_{n-1}+r$, with $r \geq 0$. Then if

$$
\left(\begin{array}{c}
t \theta_{n-1}+r-m_{\infty} \\
r+1+j
\end{array}\right) \neq 0 \quad(\bmod p)
$$

for some $j \geq 0$, then the number of $t$-fold nuclei of $w$ in $\mathrm{PG}(n, q) \backslash H_{\infty}$ is at most $(r+1+j)(q-1)$. 
Proof. If the binomial coefficient is nonzero, then $w\left(\mathrm{PG}(n, q) \backslash H_{\infty}\right)>0$, so the number of $t$-fold nuclei in $\operatorname{PG}(n, q) \backslash H_{\infty}$ is at most $q^{n}-1$. Thus the statement is trivially true for $r+1 \geq \theta_{n-1}$, so from now on we will suppose $r<\theta_{n-1}-1$.

Identify the points of $\operatorname{AG}(n, q):=\operatorname{PG}(n, q) \backslash H_{\infty}$ with the elements of $\operatorname{GF}\left(q^{n}\right)$, and the points of $H_{\infty}$ with the $\theta_{n-1}$-st roots of unity of $\operatorname{GF}\left(q^{n}\right)$ in the usual way. The points of $\operatorname{PG}(n, q)$ will be denoted by capital letters, and the corresponding elements of $\mathrm{GF}\left(q^{n}\right)$ by the same lowercase letters. Then for points $A \neq B \in \mathrm{AG}(n, q)$, the line $A B$ contains the ideal point $C \in H_{\infty}$ if and only if $(a-b)^{q-1}=c$ holds.

Let $\mathcal{S}=\left\{a_{1}, a_{2}, \ldots, a_{t \theta_{n-1}+r-m_{\infty}}\right\} \cup\left\{c_{1}, \ldots, c_{m_{\infty}}\right\}$ be the multiset of elements of $\mathrm{GF}\left(q^{n}\right)$ corresponding to the points of nonzero weight of $\operatorname{PG}(n, q) \backslash H_{\infty}$ and $H_{\infty}$ respectively, such that $a \in \mathcal{S}$ has multiplicity $w(A)$ in $\mathcal{S}$ for the corresponding point $A \in \mathrm{PG}(n, q)$.

Let $X$ and $Y$ be variables, and define

$$
\mathcal{B}(X)=\left\{\left(X-a_{i}\right)^{q-1} \mid i=1, \ldots, t \theta_{n-1}+r-m_{\infty}\right\} \cup\left\{c_{1}, \ldots, c_{m_{\infty}}\right\},
$$

and

$$
F(Y, X)=\prod_{b \in \mathcal{B}(X)}(Y-b)
$$

Then

$$
F(Y, X)=\sum_{j=0}^{t \theta_{n-1}+r}(-1)^{j} \sigma_{j}(\mathcal{B}(X)) Y^{t \theta_{n-1}+r-j},
$$

where $\sigma_{j}(\mathcal{B}(X))$ denotes the $j$ th elementary symmetric polynomial of the set $\mathcal{B}(X)$.

Suppose that $x \in \mathrm{GF}\left(q^{n}\right)$ is an element corresponding to a $t$-fold nucleus of $w$. Then $\mathcal{B}(x)$ contains every $\theta_{n-1}$-st root of unity with multiplicity at least $t$, so

$$
F(Y, x)=\left(Y^{\theta_{n-1}}-1\right)^{t}\left(Y^{r}+\text { terms of lower degree }\right) .
$$

As $r<\theta_{n-1}-1$, the coefficients of the terms

$$
Y^{t \theta_{n-1}-1}, Y^{t \theta_{n-1}-2}, \ldots, Y^{(t-1) \theta_{n-1}+r+1}
$$

are 0 in $F(Y, x)$. Thus $\sigma_{r+1+j}(\mathcal{B}(x))=0$ for $0 \leq j \leq \theta_{n-1}-r-2$. 
The degree of $\sigma_{r+1+j}(\mathcal{B}(X))$ as a polynomial of $X$ is at most $(r+1+j)(q-1)$, with equality precisely if the binomial coefficient

$$
\left(\begin{array}{c}
t \theta_{n-1}+r-m_{\infty} \\
r+1+j
\end{array}\right)
$$

does not vanish. In this case $\sigma_{r+1+j}(\mathcal{B}(X))$ is not the zero polynomial, and every nucleus is a root of it, hence the number of nuclei is at most its degree: $(r+1+j)(q-1)$.

We will now use Lemma 3.4 for $n=2, j=0$ and $m_{\infty}=t-1$.

Lemma 3.5. Suppose that $v$ is a weight function of $\mathrm{PG}(2, q)$ such that there is a line $l_{\infty}$, with $v\left(l_{\infty}\right)=t-1$, while all other lines have weight at least $t$. Then $|v| \geq(t+1) q-1$.

Proof. Assume first that $t \leq q-2$. Suppose on the contrary that $v$ is such a weight function, yet the total weight of $v$ is less than $(t+1) q-1$. We may suppose $|v|=(t+1) q-2$ (or else increase the weight of some of the points of $\left.\operatorname{PG}(2, q) \backslash l_{\infty}\right)$. All lines other than $l_{\infty}$ have weight at least $t$, which means that all the points of $\mathrm{PG}(2, q) \backslash l_{\infty}$ with weight 0 are $t$-fold nuclei of $v$. As $v\left(\mathrm{PG}(2, q) \backslash l_{\infty}\right)=(t+1) q-2-(t-1)=t q+q-t-1, \mathrm{PG}(2, q) \backslash l_{\infty}$ has at most $t q+q-t-1$ points with positive $v$ weight (and exactly this many if every point of $\operatorname{PG}(2, q) \backslash l_{\infty}$ has weight $\left.\leq 1\right)$. So $v$ has at least $q^{2}-(t q+q-t-1)=q^{2}-t q-q+t+1 t$-fold nuclei.

We will use Lemma 3.4 to prove that this is not possible. As

$$
|v|=(t+1) q-2=t(q+1)+q-t-2
$$

and

$$
\left(\begin{array}{c}
t(q+1)+q-t-2-(t-1) \\
q-t-2+1
\end{array}\right)=\left(\begin{array}{c}
t q+q-t-1 \\
q-t-1
\end{array}\right) \neq 0 \quad(\bmod p)
$$

by Lucas' theorem, so Lemma 3.4 yields that the number of $t$-fold nuclei of $v$ is at most $(q-t-1)(q-1)=q^{2}-t q-2 q+t+1$, a contradiction. The same arguments prove that, if $|v|=(t+1) q-1$, then $v(P) \leq 1$ for all points $P \in \mathrm{PG}(2, q) \backslash l_{\infty}$.

For $t \geq q-1$, the assertion can be proved by summing the weights of all lines through a carefully selected point $P$. If $P \in \mathrm{PG}(2, q) \backslash l_{\infty}$ and $v(P)=0$, 
then $|v| \geq t(q+1)=t q+t \geq t q+q-1$. If $P \in l_{\infty}$ and $v(P)=0$, then $|v| \geq t q+t-1$ and so if $t \geq q$, then we are done. If $t=q-1$ and all points of $\mathrm{PG}(2, q) \backslash l_{\infty}$ have positive weight, then $v\left(\mathrm{PG}(2, q) \backslash l_{\infty}\right) \geq q^{2}$, so $|v| \geq q^{2}+t-1>(t+1) q-1$. With this we have proved that if we can select a point $P \in \mathrm{PG}(2, q)$ with $v(P)=0$, then the assertion is true.

Assume now that $v(P)>0$ for every point, let $m=\min _{P} v(P)$ and define a new weight function $\tilde{v}$, by $\tilde{v}(P):=v(P)-m$. Then $\tilde{v}\left(l_{\infty}\right)=t-m(q+1)-1$ and $\tilde{v}(l) \geq t-m(q+1)$ for any line $l \neq l_{\infty}$. If $t-m(q+1) \leq q-2$ then we can use the first part of the proof to prove $|\tilde{v}| \geq(t-m(q+1)+1) q-1$. If $t-m(q+1) \geq q-1$ then we can use the second part, as there will be a point with zero $\tilde{v}$ weight. Then

$|v|=|\tilde{v}|+m\left(q^{2}+q+1\right) \geq(t-m(q+1)+1) q-1+m\left(q^{2}+q+1\right)=(t+1) q-1+m$.

Hence the result is established.

\section{Proof of the main theorem}

Theorem 1.3. A weighted t-fold $(n-k)$-blocking set of $\mathrm{PG}(n, q)$, with total weight smaller than

$$
(t+1) q^{n-k}+\theta_{n-k-1}
$$

contains a unique minimal weighted $t$-fold $(n-k)$-blocking set.

Proof. Assume that $w$ is a weighted $t$-fold $(n-k)$-blocking set of $\mathrm{PG}(n, q)$ which contains two different minimal $t$-fold $(n-k)$-blocking sets. We will prove $|w| \geq(t+1) q^{n-k}+\theta_{n-k-1}$. By Lemma 2.2 there is a weight function $v \leq w$, a line $l^{*}$ and a $k$-subspace $\Pi_{k}^{*}$ containing $l^{*}$, such that

(a) $v\left(\Pi_{k}\right) \geq t$, for every $k$-subspace $\Pi_{k}$ not containing $l^{*}$;

(b) $v\left(\Pi_{k}\right) \geq t-1$ for every $k$-subspace $\Pi_{k}$ containing $l^{*}$;

(c) $v\left(\Pi_{k}^{*}\right)=t-1$;

(d) $|w| \geq|v|+2$. 


\section{Case 1}

Assume first that $k=1$. Then $\Pi_{k}^{*}=l^{*}$ is a line, and $v\left(l^{*}\right)=t-1$, while the $v$ weight of any other line is at least $t$. If $n=2$, then $|v| \geq(t+1) q-1$ by Lemma 3.5, which proves the theorem in this case. Now assume $n \geq 3$ and let $\Pi$ be a plane containing the line $l^{*}$. Then the weight function $v$ restricted to the plane $\Pi$ fulfills the requirements of Lemma 3.5, so $v(\Pi) \geq$ $(t+1) q-1$. This is true for all the planes containing the line $l^{*}$, so clearly $|v| \geq \theta_{n-2} \cdot((t+1) q-1-(t-1))+t-1=(t+1) q^{n-1}+\theta_{n-2}-2$.

\section{Case 2}

For $n \geq 3$ and $k \geq 2$ we will use induction on $n$ to prove that

$$
|v| \geq(t+1) q^{n-k}+\theta_{n-k-1}-2 .
$$

Case 2a Let $V \in \Pi_{k}^{*} \backslash l^{*}$ be a point with $v(V)=0$. Consider the quotient space $\mathrm{PG}(n, q) / V \cong \mathrm{PG}(n-1, q)$, and the weight function $\tilde{v}$ induced by $v$ on $\mathrm{PG}(n-1, q)$. Clearly $\tilde{v}(\mathrm{PG}(n-1, q))=v(\mathrm{PG}(n, q))$. The plane $\left\langle V, l^{*}\right\rangle$ corresponds to a line, and a $k$-space containing $V$ corresponds to a $(k-1)$ space. It is not hard to check that $\tilde{v}$ fulfills requirements (a)-(c) with $\left\langle V, l^{*}\right\rangle / V$ as $l^{*}$ and $\Pi_{k}^{*} / V$ as $\Pi_{k-1}^{*}$, and so by induction

$$
\tilde{v}(\mathrm{PG}(n-1, q)) \geq(t+1) q^{n-k}+\theta_{n-k-1}-2 .
$$

Case 2b Suppose now that for all $P \in \Pi_{k}^{*} \backslash l^{*}: v(P)>0$, but there is a point $v(V)=0$. Then $t-1 \geq \theta_{k}-(q+1)$. Increase the weight of one point $(\neq V)$ of $l^{*}$ by one to obtain the new weight function $v^{\prime}$, which is now a $t$-fold $(n-k)$-blocking set of $\operatorname{PG}(n, q)$. We will prove that $\left|v^{\prime}\right| \geq t q^{n-k}+\theta_{n-k}-1$. This is generally not true for $t$-fold $(n-k)$-blocking sets of $\mathrm{PG}(n, q)$, only if $t$ is large enough.

Assume, on the contrary, that $\left|v^{\prime}\right| \leq t q^{n-k}+\theta_{n-k}-2$. Then we can find a line $\Sigma_{1}$ containing $V$, such that

$$
v^{\prime}\left(\Sigma_{1}\right) \leq \frac{t-\left(q^{k-1}+q^{k-2}+\cdots+q\right)}{q^{k-1}},
$$

because if all lines through $V$ had $v^{\prime}$ weight more than

$$
\frac{t-\left(q^{k-1}+q^{k-2}+\cdots+q\right)}{q^{k-1}},
$$


then all these weights would be at least $\geq \frac{t-\left(q^{k-1}+q^{k-2}+\cdots+q\right)}{q^{k-1}}+\frac{1}{q^{k-1}}$, and then the total weight of $v^{\prime}$ would be

$$
\begin{gathered}
\left|v^{\prime}\right| \geq\left(\frac{t-q^{k-1}-q^{k-2}-\cdots-q}{q^{k-1}}+\frac{1}{q^{k-1}}\right) \cdot \theta_{n-1} \\
=t q^{n-k}+\left(\frac{t}{q^{k-1}}-\frac{q^{k}+q^{k-1}+\cdots+q^{2}}{q^{k-1}}\right) \theta_{n-2}-\frac{q^{k-1}+q^{k-2}+\cdots+q}{q^{k-1}}+\frac{\theta_{n-1}}{q^{k-1}} \\
>t q^{n-k}+\frac{q^{n-1}+q^{n-2}+\cdots+q^{k}}{q^{k-1}}=t q^{n-k}+\theta_{n-k}-1 .
\end{gathered}
$$

We will now prove that if $1 \leq j \leq k-2$ and $\Sigma_{j}$ is a $j$-space with

$$
v^{\prime}\left(\Sigma_{j}\right) \leq \frac{t-\left(q^{k-j}+q^{k-j-1}+\cdots+q\right)}{q^{k-j}},
$$

then we can find a $(j+1)$-space $\Sigma_{j+1} \supset \Sigma_{j}$, with

$$
v^{\prime}\left(\Sigma_{j+1}\right) \leq \frac{t-\left(q^{k-j-1}+\cdots+q\right)}{q^{k-j-1}} .
$$

If this were not true, then we would have

$$
\begin{gathered}
\left|v^{\prime}\right|>\left(\frac{t-\left(q^{k-j-1}+\cdots+q\right)}{q^{k-j-1}}-v^{\prime}\left(\Sigma_{j}\right)\right) \cdot \theta_{n-j-1}+v^{\prime}\left(\Sigma_{j}\right) \\
\geq\left(\frac{t-\left(q^{k-j-1}+\cdots+q\right)}{q^{k-j-1}}-\frac{t-\left(q^{k-j}+q^{k-j-1}+\cdots+q\right)}{q^{k-j}}\right) \cdot \theta_{n-j-1} \\
\quad+\frac{t-\left(q^{k-j}+q^{k-j-1}+\cdots+q\right)}{q^{k-j}}=t q^{n-k}+\theta_{n-k}+1 .
\end{gathered}
$$

Thus we can find a $(k-1)$-space $\Sigma_{k-1}$, with $v^{\prime}\left(\Sigma_{k-1}\right) \leq \frac{t-q}{q}$. But all $k$-spaces containing $\Sigma_{k-1}$ have $v^{\prime}$ weight at least $t$, so

$$
\left|v^{\prime}\right| \geq\left(t-\frac{t}{q}+1\right) \cdot \theta_{n-k}+\frac{t-q}{q}=t q^{n-k}+\theta_{n-k}-1,
$$

a contradiction.

Case 2c There is one more case remaining to be proved: if $v(P)>0$ for all points $P \in \operatorname{PG}(n, q)$. Then let $m:=\min _{P} v(P)$ and let $\tilde{v}:=v-m$. Then 
$\tilde{v}$ fulfills requirements (a)-(c) with $\tilde{t}:=t-m \cdot \theta_{k}$. Cases $2 \mathrm{a}$ and $2 \mathrm{~b}$ prove $|\tilde{v}| \geq \tilde{t} q^{n-k}+\theta_{n-k}-2$ and then

$$
\begin{aligned}
|v|=|\tilde{v}|+m \cdot \theta_{n} & \geq\left(t-m \cdot \theta_{k}\right) q^{n-k}+\theta_{n-k}-2+m \cdot \theta_{n} \\
& =t q^{n-k}+\theta_{n-k}-2+m \theta_{n-k-1} .
\end{aligned}
$$

\section{$5 \quad$ Examples}

In this section we investigate the sharpness of Theorem 1.3. We are looking for weighted $t$-fold $(n-k)$-blocking sets of size $(t+1) q^{n-k}+\theta_{n-k-1}$, which contain two different minimal $t$-fold $(n-k)$-blocking sets.

\subsection{The case $t=1$}

Example 1 Let $\Pi^{1}$ and $\Pi^{2}$ be two $(n-k)$-dimensional subspaces of $\mathrm{PG}(n, q)$ meeting in an $(n-k-1)$-dimensional subspace. Then $B:=\Pi^{1} \cup \Pi^{2}$ contains two different minimal 1-fold $(n-k)$-blocking sets $\left(\Pi^{1}\right.$ and $\left.\Pi^{2}\right)$, and $|B|=$ $2 q^{n-k}+\theta_{n-k-1}$.

Corollary 5.1. Theorem 1.3 is sharp, if $t=1$.

The following proposition is a corollary of Theorem 1.3, but in fact equivalent to it if $t=1$ and $k=1$. Corollary 5.3 can also be found in [13].

Proposition 5.2. Let $B$ be a minimal 1-fold $(n-1)$-blocking set of $\mathrm{PG}(n, q)$, and $P \in B$. Then there are at least $\geq 2 q^{n-1}+\theta_{n-2}-|B|$ tangents thorugh $P$.

Proof. Suppose that there are $k$ tangents through $P$. Take points $P_{1}, P_{2}, \ldots$, $P_{k}$, one from each of the tangents, $P_{i} \neq P$. Clearly $(B \backslash\{P\}) \cup\left\{P_{1}, \ldots, P_{k}\right\}$ is a 1-fold $(n-1)$-blocking set. It contains a minimal 1-fold $(n-1)$-blocking set $B^{\prime}$, and $B \neq B^{\prime}$. Thus $B \cup\left\{P_{1}, \ldots, P_{k}\right\}$ contains two different minimal 1-fold $(n-1)$-blocking sets, so $|B|+k \geq 2 q^{n-1}+\theta_{n-2}$. 
Corollary 5.3. Let $B$ be any 1-fold $(n-1)$-blocking set of $\operatorname{PG}(n, q)$, and $P \in B$ an essential point of $B$. Then there are at least $\geq 2 q^{n-1}+\theta_{n-2}-|B|$ tangents thorugh $P$.

Construction 1 Let $B$ be a 1-fold $(n-1)$-blocking set which has a point $P \in B$, through which there are exactly $2 q^{n-1}+\theta_{n-2}-|B|$ tangents to $B$. Then adding a point to every tangent will result in a 1-fold $(n-1)$-blocking set of size $2 q^{n-1}+\theta_{n-2}$, which contains two different minimal 1-fold $(n-1)$ blocking sets.

Construction 2 Embed construction 1 in an $(n-k+1)$-dimensional subspace of $\mathrm{PG}(n, q)$ to obtain 1 -fold $(n-k)$-blocking sets of size $2 q^{n-k}+\theta_{n-k-1}$, which contain two different minimal 1-fold $(n-k)$-blocking sets.

Note that blocking sets used in the above construction exist: the so called Rédei type blocking sets always contain points which are on exactly $2 q^{n-1}+$ $\theta_{n-2}-|B|$ tangents (see [10]).

\subsection{The case $t \geq 2$}

We will use the following notation: for the multisets $B_{1}$ and $B_{2}$, with associated weight functions $w_{1}$ and $w_{2}$ respectively, $B_{1} \cup B_{2}$ will denote the multiset defined by the weight function $\max \left\{w_{1}, w_{2}\right\}$, while $B_{1}+B_{2}$ will denote the multiset defined by the weight function $w_{1}+w_{2}$.

Note that the proof of Lemma 3.5 yields that for $n=2, k=1$ it is not possible to have $v(\mathrm{PG}(2, q))=(t+1) q-1$, if $t \geq q+1$, and so the proof of Theorem 1.3 yields that the bound cannot be sharp if $t \geq q+1$. Also from the proofs of Lemma 3.5 and Theorem 1.3 it follows that if $t \leq q-2$ and $B$ is a weighted $t$-fold $(n-k)$-blocking set which contains two different minimal $t$-fold $(n-k)$-blocking sets and $|B|=(t+1) q^{n-k}+\theta_{n-k-1}$, then only points on one line (the line $l^{*}$ ) can be multiple points.

Example 2 Let $\Pi$ be a plane of $\mathrm{PG}(n, k)$, let $l_{1}, l_{2}, \ldots, l_{t}$ be different lines in $\Pi$ through a common point $P$, and $l_{t+1}$ a further line of $\Pi$, with $P \notin l_{t+1}$. Then the multiset $B:=\left(l_{1}+l_{2}+\cdots+l_{t}\right) \cup l_{t+1}$ is a $t$-fold 1-blocking set in $\mathrm{PG}(n, q),|B|=t(q+1)+(q+1-t)=(t+1) q+1$, and $l_{1}+l_{2}+\cdots+l_{t}$ and $l_{1} \cup\left(l_{2}+\cdots+l_{t}\right) \cup l_{t+1}$ are two minimal $t$-fold 1-blocking sets contained in $B$; the latter one differs from $B$ only in the point $P$. 
Corollary 5.4. Theorem 1.3 is sharp if $k=n-1,2 \leq t \leq q$.

The following proposition is again a corollary of Theorem 1.3, which is in fact equivalent to it if $k=1$. For $n=2$ and with an upper bound on the size of $B$, it can also be found in [6].

Proposition 5.5. Let $B$ be a minimal t-fold $(n-1)$-blocking set of $\mathrm{PG}(n, q)$, and $P \in B$. Then there are at least $\geq(t+1) q^{n-1}+\theta_{n-2}-|B| t$-secants through $P$.

Proof. Suppose that there are $k t$-secants through $P$. Take points $P_{1}, P_{2}, \ldots$, $P_{k}$, one from each of the $t$-secants, $P_{i} \neq P$. Clearly the $t$-fold $(n-1)$-blocking set $B \backslash\{P\}+\left\{P_{1}, \ldots, P_{k}\right\}$ contains a minimal $t$-fold $(n-1)$-blocking set $B^{\prime}$, and $B \neq B^{\prime}$. Thus $B+\left\{P_{1}, \ldots, P_{k}\right\}$ contains two different minimal $t$-fold $(n-1)$-blocking sets, so $|B|+k \geq(t+1) q^{n-1}+\theta_{n-2}$.

Construction 3 Let $B$ be a minimal $t$-fold $(n-1)$-blocking set which has a point $P \in B$, through which there are exactly $(t+1) q^{n-1}+\theta_{n-2}-|B|$ $t$-secants to $B$. Then adding a point to every $t$-secant will result in a $t$-fold $(n-1)$-blocking set of size $(t+1) q^{n-1}+\theta_{n-2}$ and containing two different minimal $t$-fold $(n-1)$-blocking sets.

Construction 4 Embed Construction 3 in an $(n-k+1)$-dimensional subspace of $\mathrm{PG}(n, q)$ to obtain $t$-fold $(n-k)$-blocking sets of size $(t+1) q^{n-k}+$ $\theta_{n-k-1}$, which contain two different minimal $t$-fold $(n-k)$-blocking sets.

For $n=2, k=1$ and $2 \leq t \leq q$ one can find $t$-fold 1-blocking sets in PG(2,q) which have points that are on exactly $(t+1) q+1-|B| t$-secants to $B$ : take the sum of $t$ Rédei type blocking sets which have a common Rédei line, and share exactly one point, that is not on the Rédei line. Example 2 is a special case of this: the sum of $t$ lines sharing a common point. Then, with Construction 4, we get examples for $n \geq 3, k=n-1$ and $1 \leq t \leq q$. Unfortunately, for $t \geq 2, n \geq 3$ and $k=1$, in the minimal $t$-fold $(n-1)$-blocking sets examined by the author all points have at least $t \theta_{n-1}-(q+1-t) q^{n-2}-|B| t$-secants to $B$. Thus it may be conjectured that the correct bound in Theorem 1.3 should be

$$
t \theta_{n-k}+(q+1-t) q^{n-k-1}
$$




\section{Acknowledgment}

I am very grateful to Leo Storme for his valuable suggestions and help.

\section{References}

[1] Ball, S.: On nuclei and blocking sets in Desarguesian spaces. J. Combin. Theory Ser. A 85, 232-236 (1999)

[2] Blokhuis, A.: On nuclei and affine blocking sets. J. Combin. Theory Ser. A 67, 273-275 (1994)

[3] Blokhuis, A.: On multiple nuclei and a conjecture of Lunelli and Sce. Bull. Belg. Math. Soc. 3, 349-353 (1994)

[4] Blokhuis, A., Wilbrink, H.A.: A characterization of exterior lines of certain sets of points in $\mathrm{PG}(2, q)$. Geom. Dedicata 23, 253-254 (1987)

[5] Brouwer, A.E., Schrijver, A.: The blocking number of an affine space. J. Combin. Theory Ser. A 24, 251-253 (1978)

[6] Ferret, S., Storme, L., Sziklai, P., Weiner, Zs.: A $t(\bmod p)$ result on weighted multiple $(n-k)$-blocking sets in $\mathrm{PG}(n, q)$. Innov. Incidence Geom. 6-7, 169-188 (2009)

[7] Gács, A., Sziklai, P., Szőnyi, T.: Two remarks on blocking sets and nuclei of planes of prime order. Des. Codes Cryptogr. 10, 29-39 (1997)

[8] Jamison, R.: Covering finite fields with cosets of subspaces. J. Combin. Theory Ser. A 22, 253-266 (1977)

[9] Lavrauw, M., Storme, L., Van de Voorde, G.: On the code generated by the incidence matrix of points and $k$-spaces in $\mathrm{PG}(n, q)$ and its dual. Finite Fields Appl. 14, 1020-1038 (2008)

[10] Storme, L., Sziklai, P.: Linear pointsets and Rédei type k-blocking sets in $\mathrm{PG}(n, q)$. J. Algebraic Comb. 14, 221-228 (2001)

[11] Sziklai, P.: Nuclei of pointsets in $\mathrm{PG}(n, q)$. Discrete Math. 174, 323-327 (1997) 
[12] Szőnyi, T.: Blocking sets in Desarguesian affine and projective planes. Finite Fields Appl. 3, 187-202 (1997)

[13] Szőnyi, T., Gács, A., Weiner, Zs.: On the spectrum of minimal blocking sets in $\mathrm{PG}(2, q)$ J. Geom. 76, 256-281 (2003)

Nóra V. Harrach

Alfréd Rényi Institute of Mathematics

Hungarian Academy of Sciences

13-15 Reáltanoda u., Budapest 1053, Hungary

e-mail: hanovi@cs.elte.hu 